Rakenteiden Mekaniikka (Journal of Structural Mechanics)

Vol. 50, No 3, 2017, pp. 141 - 145

https://rakenteidenmekaniikka.journal.fi/index

https://doi.org/10.23998/rm.65004

(c) The author(s) 2017.

Open access under CC BY-SA 4.0 license.

\title{
A posteriori analysis of classical plate elements
}

Tom Gustafsson, Rolf Stenberg ${ }^{1}$ and Juha Videman

Summary. We outline the results of our recent article on the a posteriori error analysis of $C^{1}$ finite elements for the classical Kirchhoff plate model with general boundary conditions. Numerical examples are given.

Key words: Kirchhoff plate model, $C^{1}$ elements, a posteriori error estimates.

Received 19 June 2017. Accepted 16 July 2017. Published online 21 August 2017

\section{Introduction}

The purpose of our work is to fill a gap in the literature. Surprisingly, the a posteriori error analysis for classical plate finite elements has so far only been given for the fully clamped case and a load in $L^{2}$, cf. [2]. In our recent work [1], we treated a combination of all common boundary conditions (clamped, simply supported and free). In addition, we considered the cases of point and line loads.

\section{The Kirchhoff plate problem}

We denote the deflection of the plate's midsurface by $u$, the curvature by $\boldsymbol{K}$ and the moment by $\boldsymbol{M}$, and we assume isotropic linear elasticity. Hence, it holds

$$
\boldsymbol{M}(u)=\frac{d^{3}}{12} \mathbb{C} \boldsymbol{K}(u)
$$

with

$$
\mathbb{C} \boldsymbol{A}=\frac{E}{1+\nu}\left(\boldsymbol{A}+\frac{\nu}{1-\nu}(\operatorname{tr} \boldsymbol{A}) \boldsymbol{I}\right), \quad \forall \boldsymbol{A} \in \mathbb{R}^{2 \times 2},
$$

where $d$ denotes the thickness of the plate. $E$ and $\nu$ are the Young's modulus and Poisson ratio, respectively. The strain energy for an admissible deflection $v$ is then $\frac{1}{2} a(v, v)$, with

$$
a(w, v)=\int_{\Omega} \boldsymbol{M}(w): \boldsymbol{K}(v) \mathrm{d} x=\int_{\Omega} \frac{d^{3}}{12} \mathbb{C} \boldsymbol{\varepsilon}(\nabla w): \varepsilon(\nabla v) \mathrm{d} x
$$

\footnotetext{
${ }^{1}$ Corresponding author. rolf.stenberg@aalto.fi
} 
The potential energy $l(v)$ stems from the loading, which we assume to consist of a distributed load $f \in L^{2}(\Omega)$, a load $g \in L^{2}(S)$ along the line $S \subset \Omega$, and of a point load $F$ at the point $x_{0}$, so that

$$
l(v)=\int_{\Omega} f v \mathrm{~d} x+\int_{S} g v \mathrm{~d} s+F v\left(x_{0}\right) .
$$

The total energy is thus $\frac{1}{2} a(v, v)-l(v)$, and its minimisation leads to the variational form: find $u \in V$ such that

$$
a(u, v)=l(v) \quad \forall v \in V
$$

with

$$
V=\left\{v \in H^{2}(\Omega)|v|_{\Gamma_{\mathrm{c}} \cup \Gamma_{\mathrm{s}}}=0,\left.\quad \frac{\partial u}{\partial n}\right|_{\Gamma_{\mathrm{c}}}=0\right\} .
$$

We assume that the plate is clamped on the boundary part $\Gamma_{\mathrm{c}}$, simply supported on $\Gamma_{\mathrm{s}}$, and free on $\Gamma_{\mathrm{f}}=\partial \Omega \backslash\left(\Gamma_{\mathrm{c}} \cup \Gamma_{\mathrm{s}}\right)$.

By the well-known integration by parts, we get the boundary value problem. To this end we have to recall the following quantities for a admissible displacement $v$; the normal shear force $Q_{n}(v)$, the normal and twisting moments $M_{n n}(v), M_{n s}(v)$, and the effective shear force

$$
V_{n}(v)=Q_{n}(v)+\frac{\partial M_{n s}(v)}{\partial s} .
$$

With the constitutive relationship (2), an elimination yields the plate equation for the deflection $u$ :

$$
\mathcal{A}(u):=D \Delta^{2} u=l,
$$

where the so-called bending stiffness $D$ is defined as

$$
D=\frac{E d^{3}}{12\left(1-\nu^{2}\right)} \text {. }
$$

The boundary value problem is the following.

- In the domain we have the distributional differential equation

$$
\mathcal{A}(u)=l \quad \text { in } \Omega,
$$

where $l$ is the distribution defined by (4).

- On the clamped part we have the conditions: $u=0$ and $\frac{\partial u}{\partial n}=0$ on $\Gamma_{\mathrm{c}}$.

- On the simply supported part it holds: $u=0$ and $M_{n n}(u)=0$ on $\Gamma_{\mathrm{s}}$.

- On the free part it holds: $M_{n n}(u)=0$ and $V_{n}(u)=0$ on $\Gamma_{\mathrm{f}}$.

- At the corners on the free part we have the jump condition on the twisting moment

$$
\llbracket M_{n s}(u)(c) \rrbracket=0 \quad \text { for all corners } c \in \Gamma_{\mathrm{f}} .
$$

Here and below $\llbracket \cdot \rrbracket$ denotes the jump.

We consider conforming finite element methods: find $u_{h} \in V_{h} \subset V$ such that

$$
a\left(u_{h}, v\right)=l(v) \quad \forall v \in V_{h} .
$$

The finite element partitioning is denoted by $\mathcal{C}_{h}$. We assume that mesh is such that the point load is a vertex and the line load is along edges. The edges are divided into interior edges $\mathcal{E}_{h}^{i}$, edges on $S, \mathcal{E}_{h}^{S}$, edges on the free boundary $\mathcal{E}_{h}^{f}$, and edges on the simply supported boundary $\mathcal{E}_{h}^{s}$. The local error indicators are then the following. 
- The residual on each element

$$
h_{K}^{2}\left\|\mathcal{A}\left(u_{h}\right)-f\right\|_{0, K}, \quad K \in \mathcal{C}_{h} .
$$

- The jump residuals of the normal moment along interior edges

$$
h_{E}^{1 / 2}\left\|\llbracket M_{n n}\left(u_{h}\right) \rrbracket\right\|_{0, E}, \quad E \in \mathcal{E}_{h}^{i} .
$$

- The jump residuals in the effective shear force along interior edges

$$
h_{E}^{3 / 2}\left\|\llbracket V_{n}\left(u_{h}\right) \rrbracket-g\right\|_{0, E}, \quad E \in \mathcal{E}_{h}^{S}, \quad h_{E}^{3 / 2}\left\|\llbracket V_{n}\left(u_{h}\right) \rrbracket\right\|_{0, E}, \quad E \in \mathcal{E}_{h}^{i} \backslash \mathcal{E}_{h}^{S} .
$$

- The normal moment along edges on the free and simply supported boundaries

$$
h_{E}^{1 / 2}\left\|M_{n n}\left(u_{h}\right)\right\|_{0, E}, \quad E \in \mathcal{E}_{h}^{f} \cup \mathcal{E}_{h}^{s} .
$$

- The effective shear force along edges on the free boundary

$$
h_{E}^{3 / 2}\left\|V_{n}\left(u_{h}\right)\right\|_{0, E}, \quad E \in \mathcal{E}_{h}^{f} .
$$

The error estimator is defined through

$$
\begin{aligned}
\eta^{2}= & \sum_{K \in \mathcal{C}_{h}} h_{K}^{4}\left\|\mathcal{A}\left(u_{h}\right)-f\right\|_{0, K}^{2}+\sum_{E \in \mathcal{E}_{h}^{S}} h_{E}^{3}\left\|\llbracket V_{n}\left(u_{h}\right) \rrbracket-g\right\|_{0, E}^{2}+\sum_{E \in \mathcal{E}_{h}^{i} \backslash \mathcal{E}_{h}^{S}} h_{E}^{3}\left\|\llbracket V_{n}\left(u_{h}\right) \rrbracket\right\|_{0, E}^{2} \\
& +\sum_{E \in \mathcal{E}_{h}^{i}} h_{E}\left\|\llbracket M_{n n}\left(u_{h}\right) \rrbracket\right\|_{0, E}^{2}+\sum_{E \in \mathcal{E}_{h}^{f}} h_{E}^{3}\left\|V_{n}\left(u_{h}\right)\right\|_{0, E}^{2}+\sum_{E \in \mathcal{E}_{h}^{f} \cup \mathcal{E}_{h}^{s}} h_{E}\left\|M_{n n}\left(u_{h}\right)\right\|_{0, E}^{2} .
\end{aligned}
$$

Our a posteriori estimate is the following, where the energy norm is defined as $\|\mid v\| \|=$ $a(v, v)^{1 / 2}$.

Theorem 1 There exists positive constants $C_{1}, C_{2}$, such that

$$
C_{1} \eta \leq\left\|u-u_{h}\right\| \leq C_{2} \eta \text {. }
$$

\section{Numerical examples}

In the examples, we have used the Argyris triangle. In the figures, we give the meshes for the adaptive solution of a square plate with a point and line load, and for a L-shaped domain with a free boundary for the edges sharing the re-entrant corner and simply supported along the rest of the boundary.

\section{Acknowledgements}

Funding from Tekes - the Finnish Funding Agency for Innovation (Decision number 3305/31/2015) and the Finnish Cultural Foundation is gratefully acknowledged. 

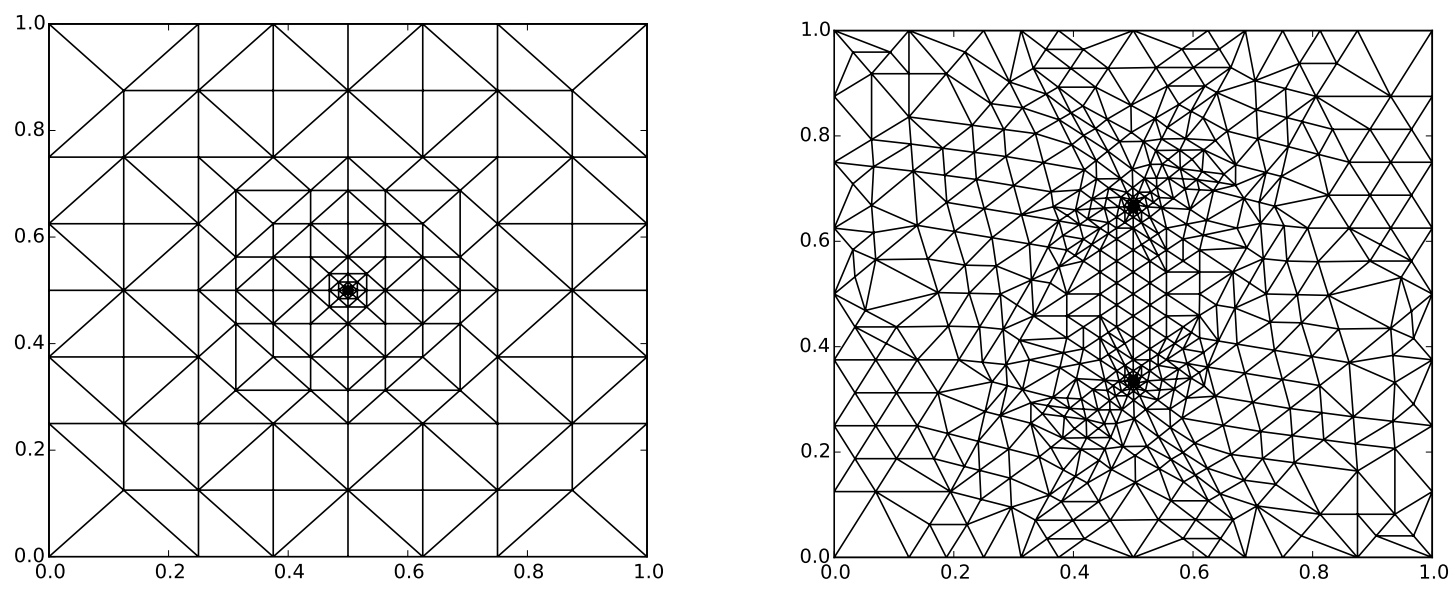

Figure 1. The adaptive meshes for the point and line loads.

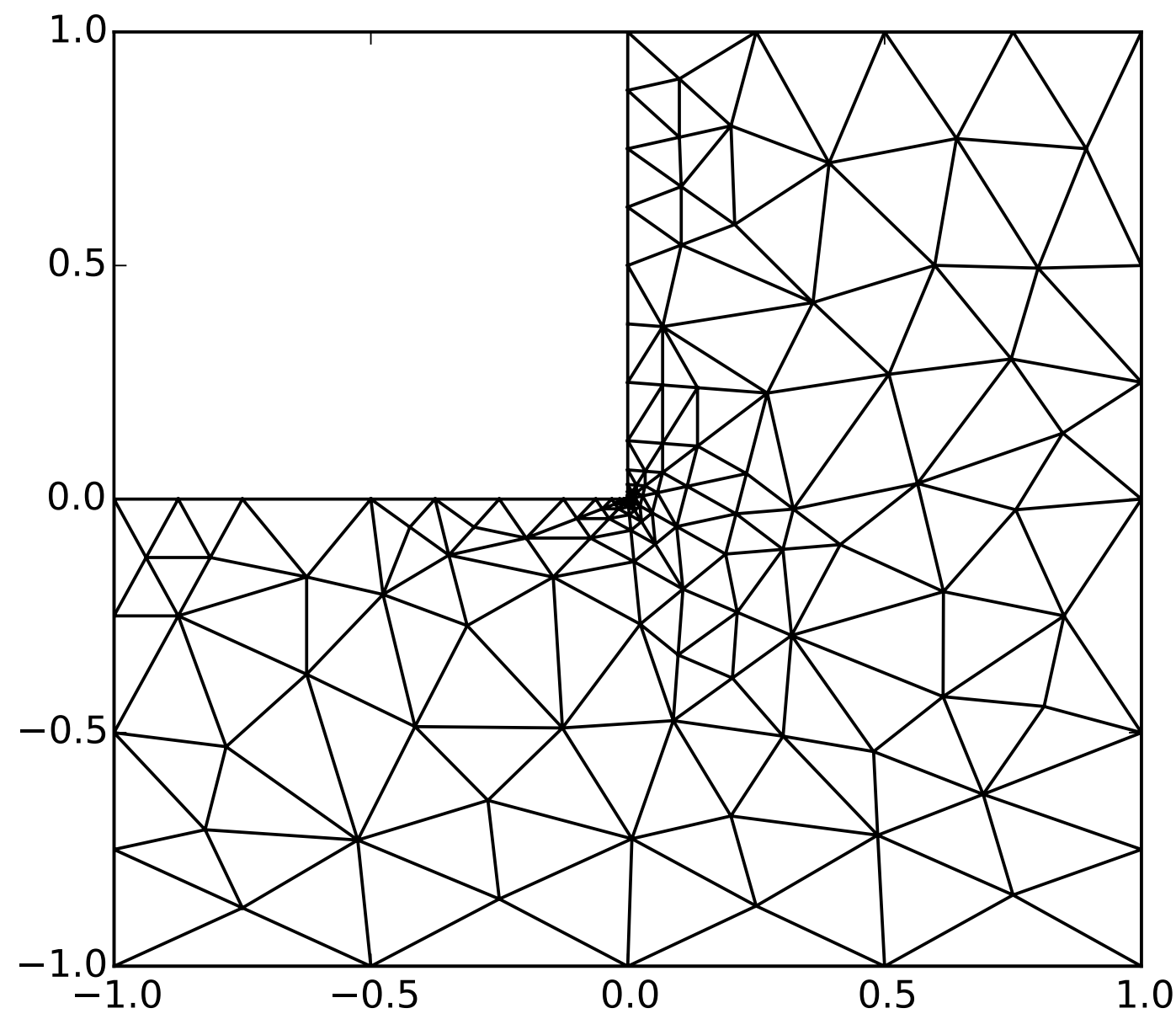

Figure 2. The adaptive mesh for the L-shaped domain. 


\section{References}

[1] Tom Gustafsson, Rolf Stenberg, and Juha Videman. A posteriori estimates for conforming Kirchhoff plate elements. arXiv:1707.08396.

[2] Rüdiger Verfürth. A posteriori error estimation techniques for finite element methods. Numerical Mathematics and Scientific Computation. Oxford University Press, Oxford, 2013.

Tom Gustafsson, Rolf Stenberg

Department of Mathematics and Systems Analysis, Aalto University - School of Science

tom.gustafsson@aalto.fi rolf.stenberg@aalto.fi

Juha Videman

CAMGSD and Mathematics Department, Instituto Superior Técnico, Universidade de Lisboa jvideman@math.tecnico.ulisboa.pt 